\title{
Forbush decrease on September 6-13, 2017 observed by the Tanca water-Cherenkov detector
}

\section{Renan de Aguiar*and Anderson Campos Fauth on behalf of the LAGO Collaboration} (a complete list of authors can be found at the end of the proceedings)

University of Campinas, Institute of Physics Gleb Wataghin,

R. Sérgio Buarque de Holanda, 777, Campinas, Brazil

E-mail: rdaguiar@ifi.unicamp.br, fauth@unicamp.br

Solar activity was intense in September 2017 and its effects were observed in different detectors placed at the Earth's surface. Three halo Coronal Mass Ejections (CME) hit the planet and caused magnetic storms. The effects of the CMEs on the flux of galactic cosmic rays at ground level were observed by the Tanca detector, which is one of the water-Cherenkov detectors (WCD) that make up the Latin American Giant Observatory (LAGO). In this paper we present the detection of Forbush events observed by Tanca during the month of September 2017. This WCD is installed on the campus of the University of Campinas, in Brazil, having three photomultiplier tubes that detect Cherenkov photons produced by cosmic radiation in 11400 liters of ultra pure water. We present the description and performance of the experimental apparatus and the observation on days $6^{\text {th }}, 8^{\text {th }}$ and $13^{\text {th }}$ of the Forbush events originated by the CMEs. A decrease in the cosmic rays flux due to a stream inTeraction region was also observed on $14^{\text {th }}$ September. These results were compared with observations made by neutron monitors and indices of the Earth's magnetic activity.

$37^{\text {th }}$ International Cosmic Ray Conference (ICRC 2021)

July 12 th - 23rd, 2021

Online - Berlin, Germany

\footnotetext{
${ }^{*}$ Presenter
} 


\section{Introduction}

The flux of galactic cosmic rays (GCR) that are detect at ground level of Earth is influenced by the solar activity, which produces perturbations on the interplanetary conditions. The Sun emits magnetized plasma (the solar wind) that propagates magnetic field into the Solar System. The conditions of the solar wind determine the level of coupling between the interplanetary medium and the magnetosphere, and can produces perturbations called geomagnetic storm. During this geomagnetic event, damage on satellites and power grids can be produced.

One solar activity that could lead to a magnetic storms is the coronal mass ejection (CME). The CME is a phenomenon where plasma and magnetic field are accelerated to several thousand kilometres per second when expelled from the corona because of large eruptions. It all starts with the increasing complexity of a closed magnetic field due to the fields continually emerging from the Sun's inner layers, until it becomes unsustainable because of the large amount of energy needed to sustain this twisting and entanglement arrangement. The result is an eruption where accumulated energy is released accelerating and heating the trapped plasma. After being released, the emitted plasma, the ejecta, propagates and, in energetic cases, produces a shock region, because, in this case, the differential velocity between the ejecta and the upstream solar wind is greater than the magnetosonic wave speed.

The consequences of the CME for the GCR flux are the know Forbush decrease (Fd). The Fd is a dropout of the cosmic ray intensity followed by a slow recover which was first described by Forbush in 1937 [1] and it is caused by both the passing of the shock and the passing of the ejecta.

There are other events that may produce the same modulation of the GCR as the CME, one of them is the stream interaction region (SIR). This structure is originated on the interaction of the high-speed stream with the preceding slower solar wind. If this structure is stable, it will co-rotate with Sun and will be called co-rotating interaction region (CIR).

September of 2017 was a non-typical period where numerous solar events were registered, such as 27M-class flares and four X-class flares [2]. Solar flares are sudden brights on the solar surface that can trigger a CME. They are split in categories according with their strength (A, B, C, M and $\mathrm{X}$ ) and in September of 2017, three flares with intensity M5.5, X9.3 and X8.2 originated three halo ${ }^{1}$ CME with the shock arrival time at 2017-09-06T23:08Z, 2017-09-07T22:30Z and 7-09-12T19:26Z, respectively [4]. These events produced Fd's that were registered by a water Cherenkov detector located University of Campinas, Brazil, called Tanca ("Tanque de Campinas" 2).

Tanca is part of LAGO (Latin American Giant Observatory) collaboration which is a network of ground based water Cherenkov detectors (WCD) spread in 9 Latin American countries in different latitudes and altitudes, covering a large range of geomagnetic rigidity cutoffs. The original objective of LAGO was to search high energy components from GRB (Gamma Ray Burst), but, in 2013, with the data provided by a several WCDs in low altitude, the collaboration started to study the solar modulations of the galactic cosmic ray and other transient events. [3]

${ }^{1}$ CMEs that propagates in direction of Earth.

${ }^{2}$ Tank of Campinas. 


\section{Methods}

The aim of Tanca is to detect, mainly, the ultrarelativistic muons that were originated by the inTeraction of the GCR with atmospheric nuclei. Tanca is a replica of the detectors used in the Pierre Auger Observatory. It is a cylinder tank with $10 \mathrm{~m}^{2}$ of base area filled with 114001 of ultra-pure and deionised water. The water is wrapped with a liner coated with Tyvek to reflect the light diffusely. At the top of the tank, there are three equidistant photomultipliers tubes (PMT) Photonics XP1805 to convert Cherenkov radiation originated from the passage of the ultrarelativistic muons in water into a measurable signal.

Figure 1 illustrates the Tanca's electronics. The negative electric pulses with variable amplitude produced by the PMTs are directed to a discriminator, Discr, where a NIM pulse ${ }^{3}$ with duration of $300 \mathrm{~ns}$ is emitted if the signal had an absolute amplitude greater than $22 \mathrm{mV}$. The discriminator also receives a signal from a pulse generator, named Clock, at a rate of $4700 \mathrm{~Hz}$ and $-800 \mathrm{mV}$ of amplitude to identify some electronic noise. Then, the signal from the discriminator goes to a logic box, Mbox A, for the search of coincidences, when two or three pulses enter the logic box in a interval of $300 \mathrm{~ns}$ after the arrival of the first pulse. Each coincidence also produces a NIM signal which are all directed to a scaler.

A complementary NIM pulse of $(1000 \pm 1) n s$, produced by one channel of a Dual Gate Generator (dgg), is plugged into the inhibit of the scaller, controlling the time that it counts the pulses.

Then, $\mathrm{C}++$ routines were set to produce a text file data containing the epoch time of measurement and the counts from the clock, each individual PMT (S1, S2 and S3), the double coincidences (D12, D13 and D23) and, finally, the triple coincidence (TRI) to every second count.

\section{Data Analyses}

The data set were filtered and the barometric effects over the data were corrected according with the formula $d \mathcal{N}=\beta d P$, where $\mathcal{N}$ is the detector count rate, $P$ is the atmospheric pressure and $\beta$ is the barometric coefficient. For the analysed period, it was measured $\beta=-(0.13 \pm 0.01) \% / \mathrm{mbar}$.

To compare and validate the Tanca's information, the time series of the detector's data were compared with other two data set from neutron monitors available in the Neutron Monitor Database (NMDB) [5]. The criteria used for the selection of the stations for comparison was to chose one station with close cuttoff rigidity with Campinas $\left(R_{C}=9.36 \mathrm{GV}\right.$ [6] $)$ and another one with more sensibility located in a region with lower $R_{C}$ in which the solar modulations are clearer in the graph.

The station with the closest cutoff rigidity listed on NMDB was the Tsumeb Neutron Monitor (Tsmb), where $R_{C}=9.15 \mathrm{GV}$. This station is a project of the of the North-West University of South Africa and is located at Tsumeb, Namibia, in an altitude of $1240 \mathrm{~m}$, it started the operation in December 1976, but currently it is offline [5]. The second chosen station was the Terre Adelie neutron monitor located at the Dumont D'Urville station (Antarctica, with $R_{C}=0.01 \mathrm{GV}$ ), it started its operations in 1967 and it is working until now.

\footnotetext{
${ }^{3}$ Nuclear Instrument Module: rectangular pulse with $-800 \mathrm{mV}$ of amplitude
} 


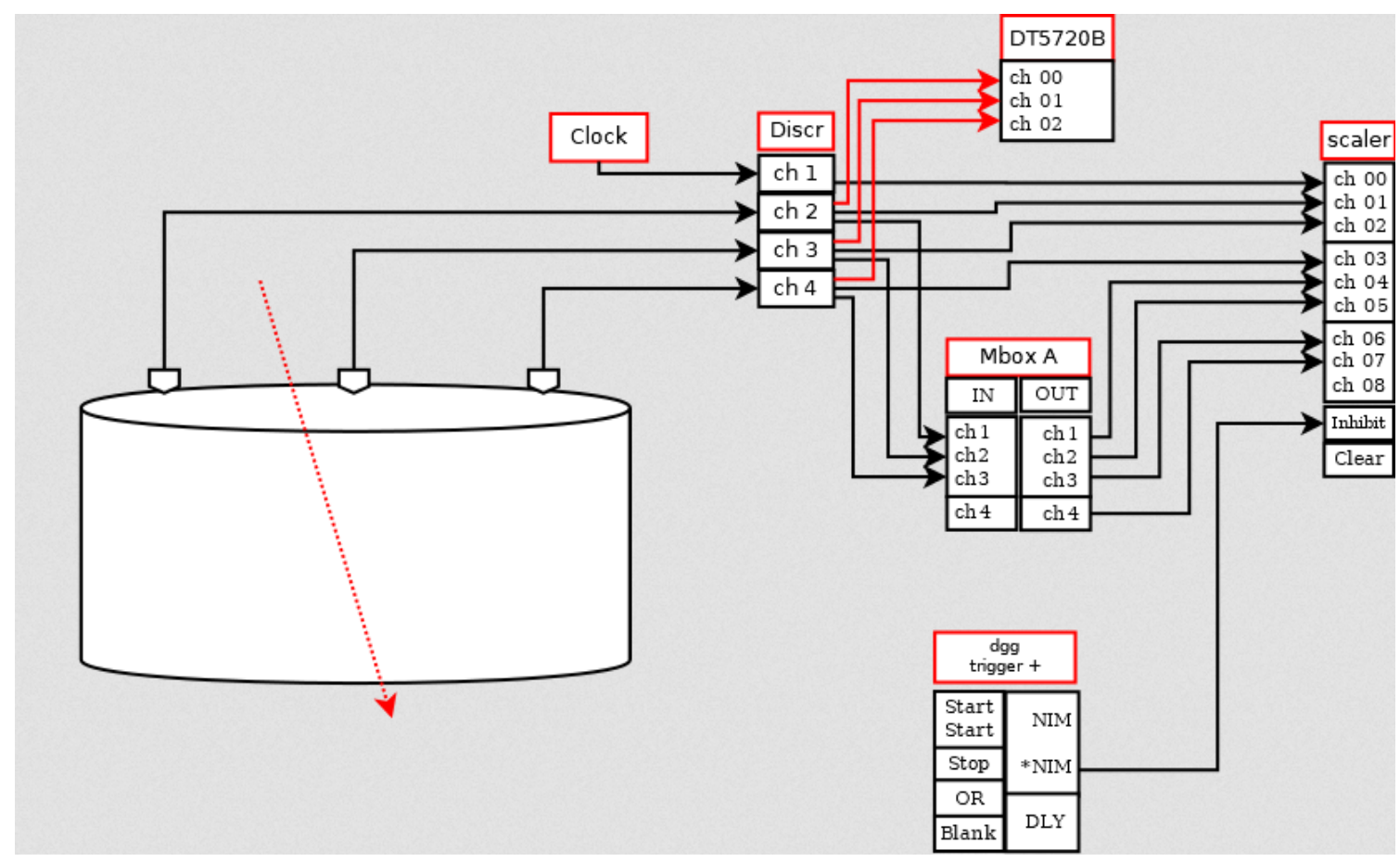

Figure 1: The data acquisition system is made with a CAMAC (Computer-Aided Measurement And Control) system, using a 8025 LeCroy rackmount mainframe and a GPIB-USAB controller. It can also be seen an illustration of a CAEN DT520B digitizer that was after installed for the study of the PMTs pulses waveforms.

\section{Results}

At Table 1 it is listed the arrival time, the geomagnetic storm parameters and the events that originated each CME. The informations are available in the CME scoreboard [9] and in SOHO LASCO CME Catalog [4].

Table 1: Halo CMEs registered by LASCO/C2 in September 2017.

\begin{tabular}{lccccc}
\hline & Arrival Time & Max Kp & Dst min.(nT) & Dst min. Time & Origin \\
\hline$C M E 1$ & $2017-09-06 \mathrm{~T} 23: 08 \mathrm{Z}$ & 4 & -23 & $2017-09-07 \mathrm{~T} 09: 00 \mathrm{Z}$ & M5.5 flare \\
$C M E 2$ & $2017-09-07 \mathrm{~T} 22: 30 \mathrm{Z}$ & 8 & -142 & $2017-09-08 \mathrm{~T} 02: 00 \mathrm{Z}$ & $\mathrm{X} 9.3$ flare \\
$C M E 3$ & $2017-09-12 \mathrm{~T} 19: 26 \mathrm{Z}$ & 5 & -50 & $2017-09-13 \mathrm{~T} 01: 00 \mathrm{Z}$ & $\mathrm{X} 8.2$ flare \\
\hline
\end{tabular}

The values of the minimum Dst related with of each CME indicate that there was a weak $(-30 \mathrm{nT}>$ Dst $>-50 \mathrm{nT})$, strong $(-100 \mathrm{nT}>$ Dst $>-200 \mathrm{nT})$ and moderate $(-50 \mathrm{nT}>$ Dst $>-100 \mathrm{nT})$ geomagnetic storm associated with the CME1, CME2 and CME3, respectively.

Figure 2 illustrates the signal detected on Tanca, Tsmb and Tera in September of 2017 . Together with the detectors information, it was also plotted the level of magnectic disturbance given by the $K p$-Index and the Dst (Disturbance Storm Time). The Kp-index is a global measurement of the geomagnetic field variation retrieved by the average of the standardised disturbance range recorded by sub-auroral stations spread around the world, while the Dst is associated with the variation of 
horizontal component of the geomagnetic field at equatorial level. The information about the Dst is provided by the World Data Center for Geomagnetism, Kyoto [8].

TANCA

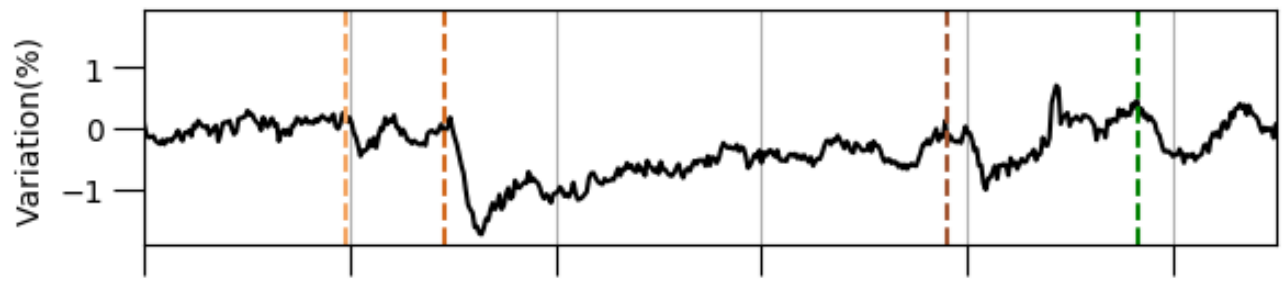

TSMB

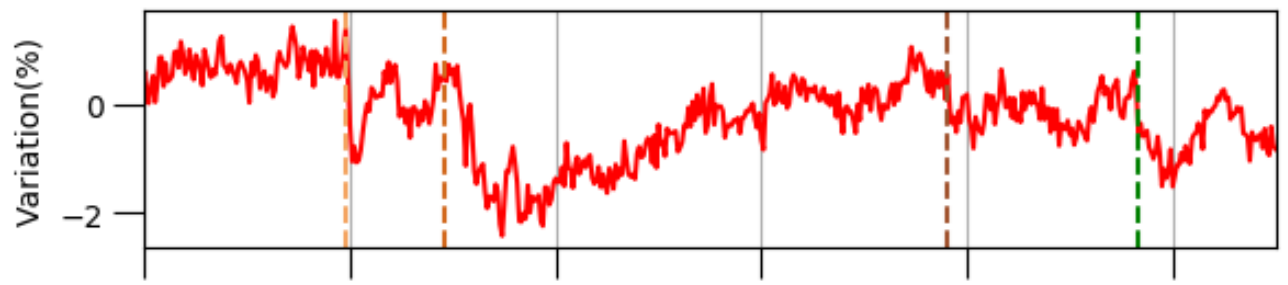

TERA

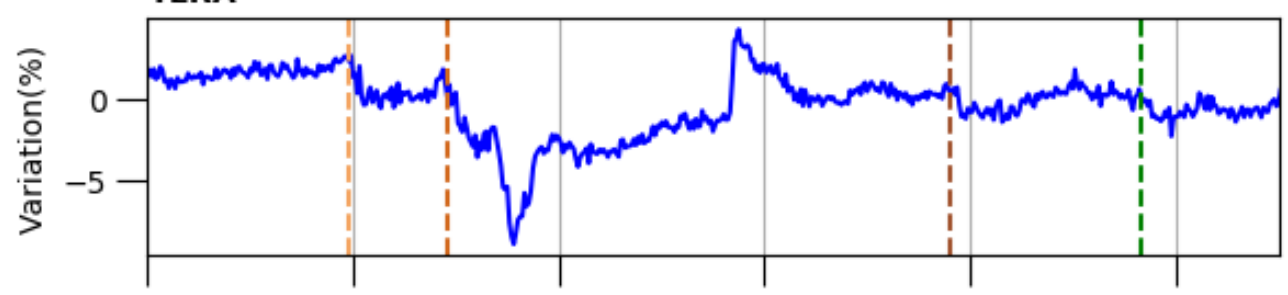

Disturbance storm - time

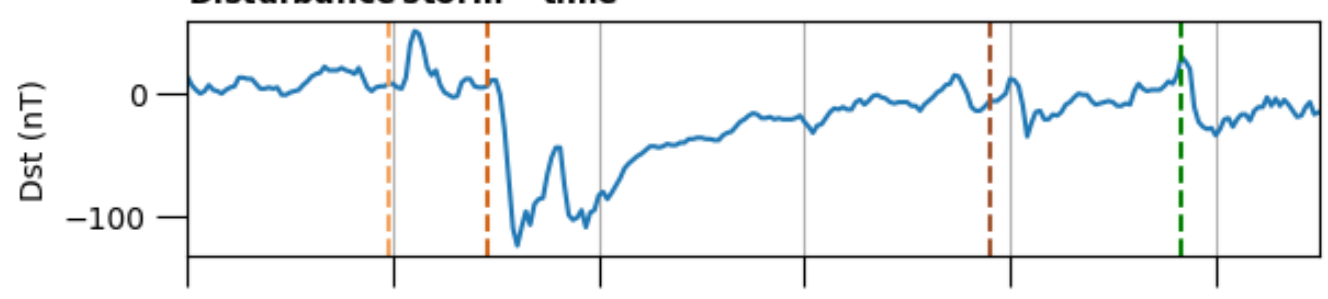

Kp index

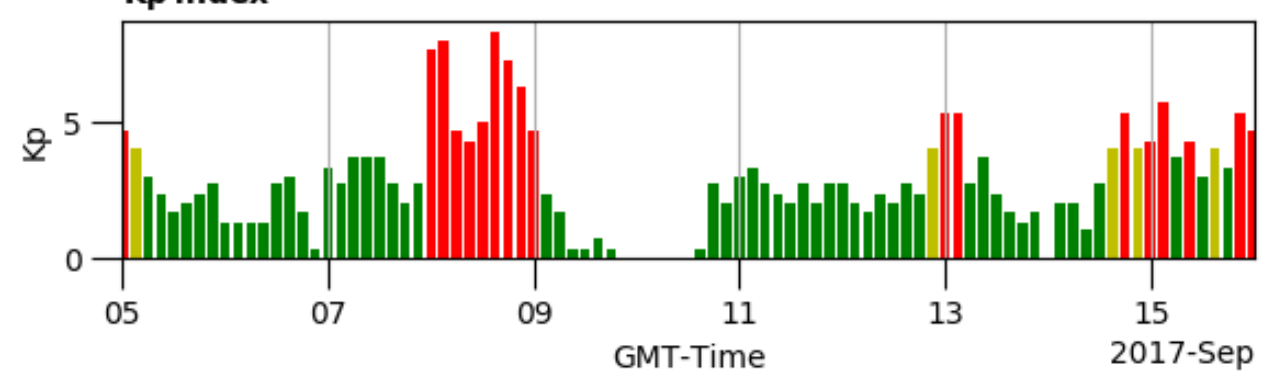

Figure 2: Cosmic ray signal from Tanca $\left(R_{C}=9.36 \mathrm{GV}\right)$ in black, Tsmb $\left(R_{C}=9.15 \mathrm{GV}\right)$ in red and Tera $\left(R_{C}=0.01 G V\right)$ in blue, hourly averaged Dst in cyan and the Kp index (green: $\mathrm{Kp}<4$, yellow: Kp $=4$ and red: $\mathrm{Kp}>4$ ) registered during the strong solar activity in September 2017. The four dashed vertical lines are associated with the shock time arrival of the CME1, CME2 and CME3, and with the SIR.

The first CME produced an immediate drop in signals from the three detectors and, after this 
first decrease, the signal intensity remained reduced until the second CME. In Tanca and Tera, the decrease caused by CME1 was less sharply in compare with the Tsmb signal. However, in Tsmb there was a peak in September $7^{\text {th }}$ which is also apparent in Tanca, but it is not present in the Tera data. The interesting feature about this peak is that it follows the positive variation of the Dst index.

With the CME2, all signals presented intense dropouts, but the decrease on Tsmb was not instantaneous, it just happened at the beggining of September $8^{\text {th }}$. On the other hand, Tanca and Tera signal started to fall approximately at the same time that the CME arrived. Tanca reached its minimum in a shorter time while comparing with the neutron monitors, probably due the fact that it mainly detects muons.

One effect that is just clear on Tera was the two steps decrease with the passage of the CME2. This two step decrease is not present on Tanca and Tsmb, where their minimum happened at the same time when the Tera completed its first decrease. Probably, the responsible for these differences is the higher cuttof rigidity in the Tanca and Tsmb experiment sites.

The recover after the second Fd is also different for each data set. While Tanca and Tsmb presented a smooth recover that ended at about September $11^{\text {th }}$, the recover of Tera had a first fast increase right after the Fd followed by a period of smooth increase until the GLE (ground level enhancement) event at September $10^{\text {th }}$. A GLE is an increase of the cosmic ray flux measured by ground based detectors, caused by the arrival of solar energetic particles associated with large solar flares. However, these particles usually do not have enough energy to reach regions with high cutoff rigidity, which explains why the GLE is invisible at Tanca and Tsmb curves.

Then, there is the effect of the arrival of the third CME at September $11^{\text {th }}$. In contrast to the previous Fds, the Tsmb signal had the faster response to the shock, while it took a while for the signals from the other detectors to drop. This Fd is the one with the biggest difference in response time between the signals, in addition, even though CME3 is associated with a moderate geomagnetic storm, the intensity of the decreases in Tsmb and Tera are clearly less intense than the decrease during the weak storm caused by CME1.

In addition to the drop in signal due to the arrival of CMEs, the Dst and $\mathrm{Kp}$ index indicated that there was a disturbance in the geomagnetic structure (Kp $>5$ and Dst $\leq-30 \mathrm{nT})$ on September $14^{\text {th }}$. At this date time, it is also noticeable a decrease on Tanca signal that was driven by the stream inTeraction regions (SIR), which is in accordance with what was recorded in other works (check Reference [10]). The Tsmb, like Tanca, showed an intense drop in signal with the SIR, in both, this dropout was more intense than that caused by CME3. However, the decrease in Tera signal was almost unnoticeable.

\section{Conclusion}

In September 2017, strong solar events in a short period of time driven to three coronal mass ejections with arrival time to Earth on September $6^{\text {th }}, 7^{\text {th }}$ and $12^{\text {th }}$. CMEs causes the phenomenon known as Forbush decrease, characterised by an abrupt drop on the cosmic ray intensity followed by a slow recover. In Tanca data, there are three Forbush decreases registered in the period in which the most evident was the one related to the second CME, and a decrease in intensity was also recorded at a time coinciding with the SIR event (September $14^{\text {th }}$ ). 
These observations of Forbush events carried out by the Tanca detector show the effects of solar events on the Earth's magnetic field for a region of energy above the energies observed by space missions, adding information about the effects of the more energetic particles of these events.

\section{Acknowledgements}

The author would like to express the gratitude for the support and sponsorship provided by the Ministry of Science, Technology and Innovation" and the "National Council for Scientific and Technological Development - CNPq" (process 131552/2019-8) and by FAEPEX (process 2399/21). We acknowledge the NMDB database (www.nmdb.eu), founded under the European Union's FP7 programme (contract no. 213007) for providing data. We also acknowledge the Observatoire de Paris and the French polar institute (IPEV), France, and the Tsumeb Geophysical Station, Namibia, for kindly providing the data from the Terre Adelie neutron and the Tsumeb station, respectively. The LAGO Collaboration is very thankful to the Pierre Auger Collaboration for their continuous support.

\section{References}

[1] S. E. Forbush. "On the Effects in Cosmic-Ray Intensity Observed During the Recent Magnetic Storm”. In: Physical Review 51.12 (1937), pp. 1108-1109. doi: 10.1103/physrev.51.1108.3.

[2] I. M. Chertok, A. V. Belov, and A. A. Abunin. "Solar Eruptions, Forbush Decreases, and Geomagnetic Disturbances From Outstanding Active Region 12673”. In: Space Weather 16.10 (2018), pp. 1549-1560. doi: 10.1029/2018sw001899.

[3] I. Sidelnik [LAGO], PoS ICRC2015 (2016), 665 doi:10.22323/1.236.0665

[4] SOHO LASCO CME CATALOG - CDAW DATA CENTER. url: https://cdaw.gsfc.nasa.gov/CME_list/

[5] Real-Time Database for high-resolution Neutron Monitor measurements. url: http : / / www01.nmdb.eu/.

[6] Cuttof Rigidity Calculator. url: http://cosmos.hwr.arizona.edu/Util/computeCR.php.

[7] CERCLe. url:https://previ.obspm.fr/

[8] World Data Center for Geomagnetism, Kyoto. url:http://wdc.kugi.kyoto-u.ac.jp/index.html.

[9] CME Scoreboard. url: https://kauai.ccmc.gsfc.nasa.gov/CMEscoreboard/.

[10] Jingnan Guo et al. "Modeling the Evolution and Propagation of 10 September 2017 CMEs and SEPs Arriving at Mars Constrained by Remote Sensing and In Situ Measurement". In: Space Weather 16.8 (2018), pp. 1156-1169. doi: 10.1029/2018sw001973. 


\section{Full Authors List: LAGO Collaboration}

V. Agosín ${ }^{20}$, A. Alberto $^{3}$, C. Alvarez ${ }^{16}$, J. Araya ${ }^{20}$, R. Arceo ${ }^{16}$, O. Areso $^{13}$, L. H. Arnaldi ${ }^{2}$, H. Asorey ${ }^{14,7}$, M. Audelo ${ }^{9}$, M.G. BallinaEscobar $^{19}$, D. C. Becerra-Villamizar ${ }^{18}$, X. Bertou ${ }^{2}$, K.S. Caballero-Mora ${ }^{16}$, R. Caiza ${ }^{8}$, R. Calderón-Ardila ${ }^{14}$, Calle, J. ${ }^{24}$, A. C. Fauth ${ }^{27}$, E. Carrera Jarrin ${ }^{26}$, L. E. Castillo Delacroix ${ }^{11}$, C. Castromonte ${ }^{25}$, Cazar-Ramírez D. ${ }^{26}$, Diego Cogollo ${ }^{28}$, D. A. Coloma Borja ${ }^{26}$, R. Conde $^{1}$, J. Cotzomi ${ }^{1}$, D. Dallara ${ }^{11}$, S. Dasso ${ }^{13,5,6}$, R. Aguiar ${ }^{27}$, Albuquerque, A. ${ }^{28}$, J.H.A.P.Reis ${ }^{27}$, H. De León ${ }^{16}$, R. deLeón-Barrios ${ }^{23}$, D. Domínguez ${ }^{8}$, M. Echiburu ${ }^{21}$, M. González², M. Gómez Berisso ${ }^{2}$, J. Grisales Casadiegos ${ }^{23}$, A. M. Gulisano ${ }^{13,12,6}$, Juan Carlos Helo $^{17}$, Condori, C. A. H. ${ }^{24}$, J. E. Ise ${ }^{11}$, Nascimento, G. K. ${ }^{28}$, M. A. Leigui de Oliveira ${ }^{29}$, F. L. Miletto ${ }^{27}$, V. P. Luzio ${ }^{29}$, F. Machado ${ }^{25}$, Juan F. Mancilla-Caceres ${ }^{22}$, D. Manriquez ${ }^{20}$, A. Martínez-Méndez ${ }^{23}$, O. Martinez ${ }^{1}$, R. Mayo-García ${ }^{3}$, L.G. Mijangos ${ }^{22}$, Miranda, $\mathrm{P}^{24}$, M. G. Molina ${ }^{11}$, I.R. Morales ${ }^{19}$, O.G Morales-Olivares ${ }^{16}$, E. Moreno-Barbosa ${ }^{1}$, P. Muñoz ${ }^{17}$, Nina, $\mathrm{C}^{24}$, L.A. Núñez ${ }^{23}$, L. Otininano ${ }^{4}$, R.Pagán $^{3}$, K. M. Parada-Jaime ${ }^{18}$, H. M. Parada-Villamizar ${ }^{18}$, R. Parra ${ }^{10}$, J. Peña-Rodríguez ${ }^{23}$, M. Pereira ${ }^{13}$, Y. A. Perez-Cuevas ${ }^{18}, H_{\text {. }}$ Perez $^{19}$, J. Pisco-Guabave ${ }^{23}$, Raljevic, M. ${ }^{24}$, M. Ramelli1 ${ }^{13}$, C. Ramírez ${ }^{22}$, Rivera, $\mathrm{H}^{24}$, L. T. Rubinstein ${ }^{13}$, A.J. Rubio-Montero ${ }^{3}$, J.R. Sacahui $^{19}$, H. Salazar ${ }^{1}$, N. Salomón ${ }^{11}$, J. Samanes ${ }^{4}$, N.A. Santos ${ }^{5}$, C. Sarmiento-Cano ${ }^{14}$, I. Sidelnik ${ }^{2}$, Mayra B. Silva ${ }^{22}$, O. Soto ${ }^{17}$, M. Suárez-Durán ${ }^{18,31}$, Subieta Vasquez, M. ${ }^{24}$, Terrazas C. ${ }^{24}$, Ticona, R. ${ }^{24}$, T. Torres Peralta ${ }^{11}$, Pablo A. Ulloa ${ }^{17}$, Z.R. Urrutia ${ }^{22}$, N. Vásquez $^{8}$, A. Vázquez-Ramírez ${ }^{23}$, A. Vega ${ }^{20}$, P. Vega ${ }^{17}$, J. Vega $^{4}$, A. Vesga-Ramirez ${ }^{14}$, D. Vitoreti ${ }^{30}$, R. Wiklich Sobrinho ${ }^{29}$,

${ }^{1}$ Benemérita Universidad Autónoma de Puebla. ${ }^{2}$ Centro Atómico Bariloche (CNEA/CONICET/IB). ${ }^{3}$ CIEMAT". ${ }^{4}$ Comisión Nacional de Investigación y Desarrollo Aeroespacial. ${ }^{5}$ Departamento de Ciencias de la Atmósfera y los Océanos, Facultad de Ciencias Exactas y Naturales, Universidad de Buenos Aires.. ${ }^{6}$ Departamento de Física (FCEN,UBA)". ${ }^{7}$ Departamento Física Médica, CNEACONICET-UNSAM. ${ }^{8}$ Escuela Politécnica Nacional. ${ }^{9}$ Escuela Superior Politécnica de Chimborazo. ${ }^{10}$ European Soutern Observatory (ESO). ${ }^{11}$ Facultad de Ciencias Exactas y Tecnología (FACET) - Universidad Nacional de Tucumán (UNT). ${ }^{12}$ Instituto Antártico Argentino, Dirección Nacional del Antartico, Instituto de Astronomía y Física del Espacio (UBA-CONICET). ${ }^{13}$ Instituto de Astronomía y Física del Espacio, IAFE (UBA-CONICET). ${ }^{14}$ Instituto de Tecnologías en Detección y Astropartículas (CNEA, CONICET,UNSAM). ${ }^{16}$ Universidad Autónoma de Chiapas. ${ }^{17}$ Universidad de La Serena. ${ }^{18}$ Universidad de Pamplona. ${ }^{19}$ Universidad de San Carlos. ${ }^{20}$ Universidad de Valparaíso. ${ }^{21}$ Universidad de Viña del Mar. ${ }^{22}$ Universidad del Valle de Guatemala. ${ }^{23}$ Universidad Industrial de Santander. ${ }^{24}$ Universidad Mayor de San Andrés. ${ }^{25}$ Universidad Nacional de Ingeniería. ${ }^{26}$ Universidad San Francisco de Quito. ${ }^{27}$ Universidade Estadual de Campinas. ${ }^{28}$ Universidade Federal de Campina Grande. ${ }^{29}$ Universidade Federal do ABC. ${ }^{30}$ Universidade Federal do Recôncavo da Bahia. ${ }^{31}$ Université Libre de Bruxelles, Brussels, Belgium.. 